\title{
Endoscopic retrieval of a distally migrated stent using detachable snares
}

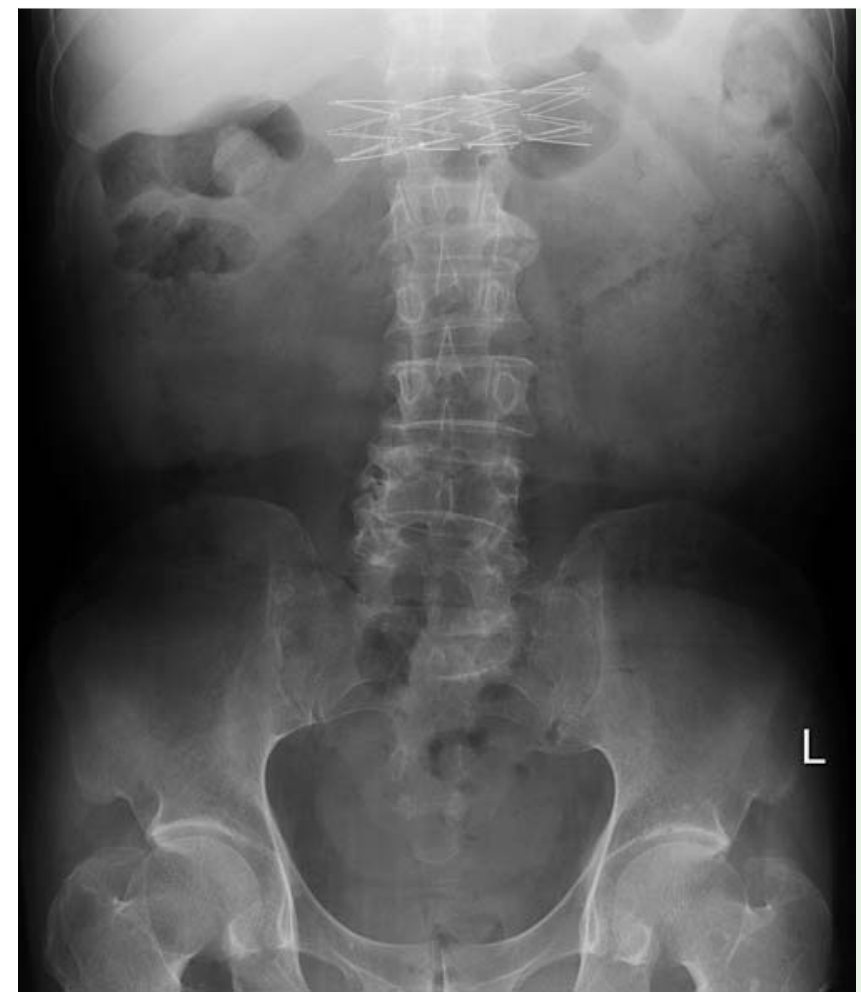

Fig. 1 The dislodged stent within the stomach shadow on abdominal X-ray.

We herein report an endoscopic method utilizing detachable snares for the safe removal of dislodged esophageal metal stents.

A 63-year-old man was diagnosed as having carcinoma of the gastroesophageal junction in 2008. Staging computed tomography (CT) confirmed hepatic and pulmonary metastases. The patient was initially treated with palliative chemotherapy, and, in 2009, a self-expandable metal esophageal stent was inserted for the worsening dysphagia. In January 2010 , the patient presented with repeated vomiting and intolerance to oral intake. An abdominal X-ray showed stent dislodgment within the stomach shadow (๑ Fig. 1).

Esophagogastroscopy revealed a partially obstructing, 9-cm long tumor at the esophagogastric junction, through which the endoscope passed with difficulty. The previously inserted stent was visualized within the stomach ( Fig. 2).

Detachable snares were applied to collapse the migrated stent inside the stomach ( Fig. $\mathbf{3 a}$ ), and a new covered metallic stent was inserted at the tumor site.
After 5 days, repeat endoscopy showed the new esophageal stent had fully deployed. The dislodged stent in the stomach was further reduced in size with detachable snares ( Fig. $\mathbf{3 b}$ ).

It was then drawn into the new stent using two endograsping forceps and the whole complex was retrieved orally under fluoroscopy monitoring ( $\bullet$ Fig.4).

A new self-expanding metal stent (SEMS) was inserted for relief of dysphagia, and the patient was then able to tolerate oral feeding. He was discharged 10 days after the second endoscopy.

Migration of SEMS has a reported incidence of up to $12 \%$ after stent insertion [1]. Extraction of the migrated stent is advocated due to the potential risks of bleeding and bowel perforation and obstruction [2]. However, endoscopic retrieval of a stent can be technically difficult in patients with obstructing esophageal tumors and is associated with the risk of tumor laceration, bleeding, and esophageal perforation. Collapsing the migrated stent by using detachable snares made retrieval relatively easy and safe in our patient.

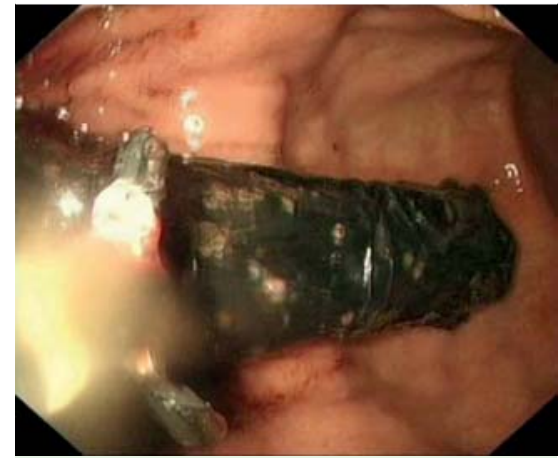

Fig. 2 The dislodged self-expandable metal stent inside the stomach.
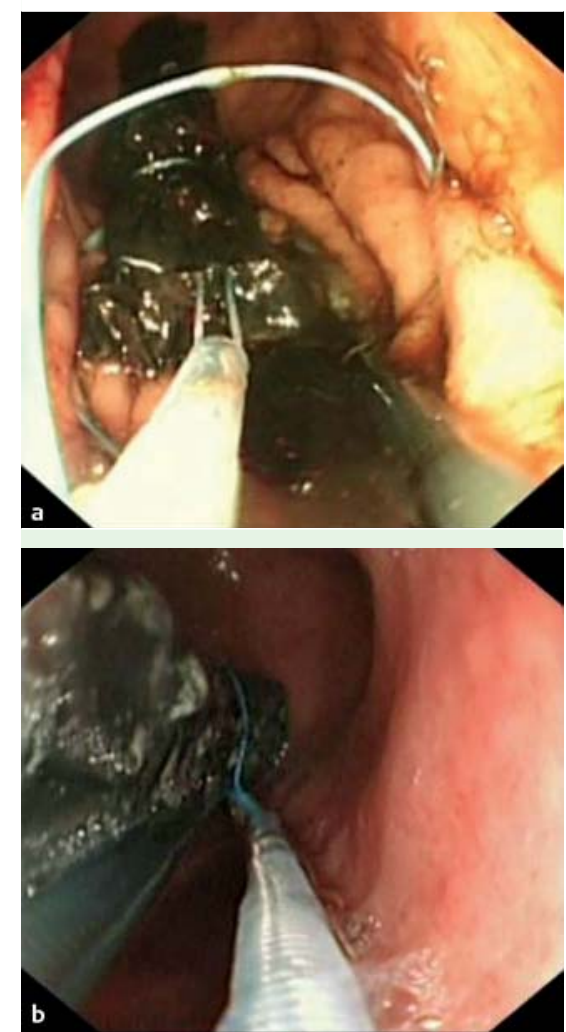

Fig. 3 Stent being collapsed with the use of detachable snares. a First attempt. b Second attempt after 5 days.

Endoscopy_UCTN_Code_TTT_1AO_2AZ

Competing interests: None 


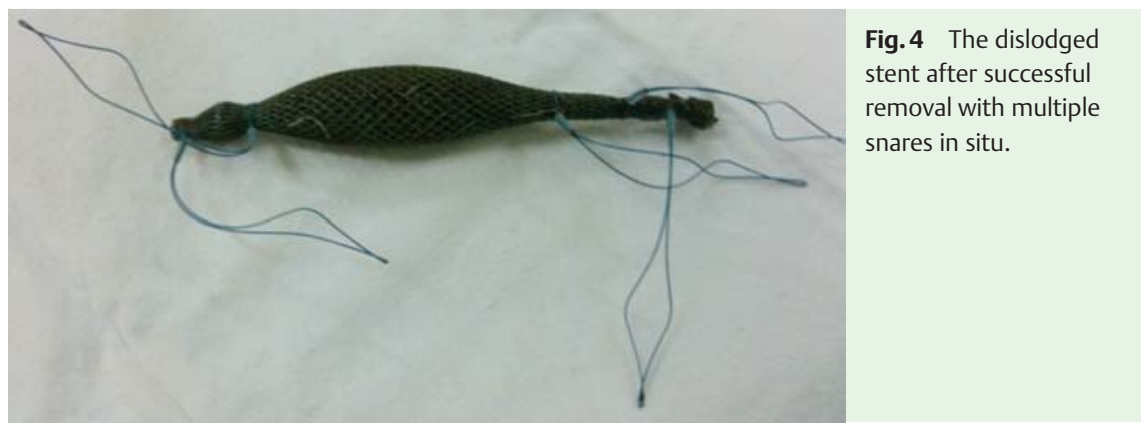

\section{H. C. Yip, P. W. Y. Chiu, E. K. W. Ng}

Department of Surgery, Prince of Wales Hospital, The Chinese University of Hong Kong, Shatin, Hong Kong SAR

\section{Bibliography}

DOI http://dx.doi.org/ 10.1055/s-0030-1256586

Endoscopy 2013; 45: E420-E421

(C) Georg Thieme Verlag KG

Stuttgart · New York

ISSN 0013-726X

\section{Corresponding author}

Professor E. K. W. Ng

Department of Surgery

Faculty of Medicine

The Chinese University of Hong Kong Shatin

Hong Kong SAR

Fax: (852)-2632-7974

endersng@surgery.cuhk.edu.hk 